04

\title{
Стримеры при субнаносекундном пробое аргона и азота в неоднородном электрическом поле при обеих полярностях
}

\author{
(C) Д.В. Белоплотов, ${ }^{1,2}$ М.И. Ломаев, ${ }^{1,2}$ Д.А. Сорокин, ${ }^{1,2}$ В.Ф. Тарасенко ${ }^{1,2}$ \\ ${ }^{1}$ Институт сильноточной электроники СО РАН, \\ 634055 Томск, Россия \\ ${ }^{2}$ Национальный исследовательский Томский государственный университет, \\ 634050 Томск, Россия \\ e-mail: rff.qep.bdim@gmail.com
}

(Поступило в Редакцию 17 августа 2017 г.)

C помощью ICCD-камеры исследовано свечение плазмы на этапе формирования стримера (волны ионизации) в промежутке „острие-плоскость“ длиной $3 \mathrm{~mm}$, заполненном аргоном либо азотом при давлении $12.5-400 \mathrm{kPa}$. На промежуток подавались наносекундные импульсы напряжения отрицательной и положительной полярности. Получены изображения стримера в различные моменты времени при его распространении по промежутку. Посредством стрик-камеры, оснащенной монохроматором, зарегистрирован временной ход интенсивности излучения молекул азота на полосе $337.1 \mathrm{~nm}$ из различных зон вдоль промежутка при отрицательной полярности. По полученным данным оценена средняя скорость стримера при атмосферном давлении азота $-1.8 \mathrm{~cm} / \mathrm{ns}$. Зарегистрированы амплитудно-временные характеристики напряжения, тока разряда и пучка убегающих электронов за анодном из алюминиевой фольги толщиной $10 \mu \mathrm{m}$. Обсуждены причины формирования диффузного разряда в данных условиях.

DOI: 10.21883/JTF.2018.06.46032.2465

\section{Введение}

В последние годы возрос интерес к пробою различных газов атмосферного давления в неоднородном электрическом поле наносекундными импульсами высокого напряжения [1-29]. В этих условиях при подаче на промежуток импульсов напряжения амплитудой $\sim 100 \mathrm{kV}$ и более регистрируются рентгеновское излучение и пучки убегающих электронов субнаносекундной длительности за анодом, изготовленным из фольги (см., например, коллективную монографию [20], в которой опубликованы результаты исследований различных научных групп, а также ссылки в ней). Отметим, что рентгеновское излучение было зарегистрировано при более низких напряжениях на разрядном промежутке, чем пучки убегающих электронов, благодаря его большей проникающей способности [9,12,16,19,20,30].

В ряде работ были проведены исследования динамики свечения плазмы в промежутке [3,6,8,10,11,13,17,21,24]. Так, в работе [3] было показано, что в воздухе атмосферного давления при положительной полярности импульсов напряжения $\left(U=43 \mathrm{kV}, \tau_{0.5}=12 \mathrm{~ns}\right.$, $\left.\tau_{0.1-0.9}=2-3 \mathrm{~ns}\right)$, подаваемых на острийный электрод, свечение плазмы возникает вблизи острийного электрода и имеет форму, близкую к шару. Диаметр светящейся области в течение нескольких наносекунд возрастал до $8 \mathrm{~mm}$ при длине промежутка $16 \mathrm{~mm}$. Излучение плазмы имело наибольшую интенсивность вблизи острия и через $2.5 \mathrm{~ns}$ занимало примерно половину разрядного промежутка. Далее фронт свечения сужался и при соприкосновении с катодом имел размер около $2 \mathrm{~mm}$. При увеличении давления до $200 \mathrm{kPa}$ и более разряд формировался в виде отдельных диффузных каналов малого диаметра, которые авторы работы [3] называли филаментами. В работе [24] исследования проводились при амплитуде импульса напряжения до $74 \mathrm{kV}$ и с бо́льшим временным разрешением. На начальной стадии разряда при атмосферном давлении также наблюдалась шарообразная форма плазмы вблизи острийного электрода. Из моделирования в настоящей работе следовало, что вблизи острийного электрода формируется стример, на фронте которого напряженность электрического поля превышает пороговое значение $(28 \mathrm{kV} / \mathrm{cm})$ для пробоя воздуха атмосферного давления. Однако ни пучок убегающих электронов, ни рентгеновское излучение в работах [2,24] не регистрировались.

Высокоскоростные волны ионизации в воздухе атмосферного давления регистрировались в работе [11] при амплитуде импульса напряжения положительной полярности, прикладываемого к промежутку, $112 \mathrm{kV}$, длительности импульса $0.4 \mathrm{~ns}$ и времени нарастания $0.15 \mathrm{~ns}$. В этом случае вблизи острийного электрода также формировался стример, который на начальном этапе имел форму шара и распространялся в направлении к плоскому электроду со скоростью более $2 \mathrm{~cm} / \mathrm{ns}$. При приближении к катоду форма стримера, как и в работе [3], изменялась, его фронт становился вытянутым. В работе [11] отмечалось, что в данных условиях достигалось критическое значение напряженности электрического поля для генерации убегающих электронов. Однако эксперименты по регистрации пучка убегающих электронов или рентгеновского излучения не ставились, по-видимому, из-за применения импульсов напряжения положительной полярности. 
В работе [10] исследование динамики свечения в промежутке проводилось при амплитуде импульса напряжения отрицательной полярности 100 и $200 \mathrm{kV}$ и времени нарастания 1 и $5 \mathrm{~ns}$ соответственно. В качестве катода использовалось бритвенное лезвие длиной $10 \mathrm{~mm}$, а анод был плоским. Свечение плазмы в этих условиях также возникало у электрода с малым радиусом кривизны (лезвие), но имело форму отдельных диффузных каналов (стримеров) сравнительно малого диаметра. Отметим, что в настоящей работе благодаря высокому напряжению $(100$ и $200 \mathrm{kV})$, а также отрицательной полярности было зарегистрировано тормозное рентгеновское излучение. Измерения пучка убегающих электронов в настоящей работе не проводились.

Пучок убегающих электронов за анодном, изготовленным из фольги, был зарегистрирован с помощью коллектора в работах $[1,7,9,21,23]$ при амплитуде импульса напряжения $>100 \mathrm{kV}$ и применения катода в виде трубки. Разряд при атмосферном давлении воздуха и азота состоял из отдельных диффузных каналов. В работе [17] несколько диффузных каналов, стартующих с катода, были зарегистрированы при напряжении в падающей волне $\approx 15 \mathrm{kV}$, длительности импульса $4-5 \mathrm{~ns}$ и времени нарастания $2-3 \mathrm{~ns}$ как с трубчатым катодом, изготовленным из фольги, так и с катодом в виде острия. При уменьшении давления азота до единиц-десятков $\mathrm{kPa}$ на подобных установках были зарегистрированы не только рентгеновское излучение, но и пучок убегающих электронов $[15,19]$.

Однако закономерности развития ионизационных процессов в промежутке при длительности предпробойной стадии $\sim 1$ ns и менее изучены на настоящее время слабо. В частности, мало экспериментальных данных о влиянии полярности импульса напряжения на формирование плазмы в промежутке „острие-плоскость“. Кроме того, нет единой точки зрения о механизме формирования диффузных разрядов в неоднородном электрическом поле в воздухе атмосферного давления и других газах при высоких перенапряжениях. Из теоретических работ (см., например, $[2,26,29])$, следует, что формирование и движение стримера обеспечивается за счет фотоионизации газа. В моделях разряд инициируется фоновыми электронами [31], а в электроотрицательных газах за счет отлипания электронов от положительных ионов. С другой стороны, в работах $[1,20,21,25,32]$ утверждается, что при отрицательной полярности импульса напряжения предварительная ионизация газа перед стримером (волной ионизации) обеспечивается быстрыми электронами. В работах $[25,33]$ предполагается, что при положительной полярности импульса напряжения начальные электроны перед фронтом волны ионизации создаются благодаря характеристическому рентгеновскому излучению.

Цель настоящей работы - получить экспериментальные данные о структуре стримера в условиях субнаносекундного пробоя и генерации убегающих электронов

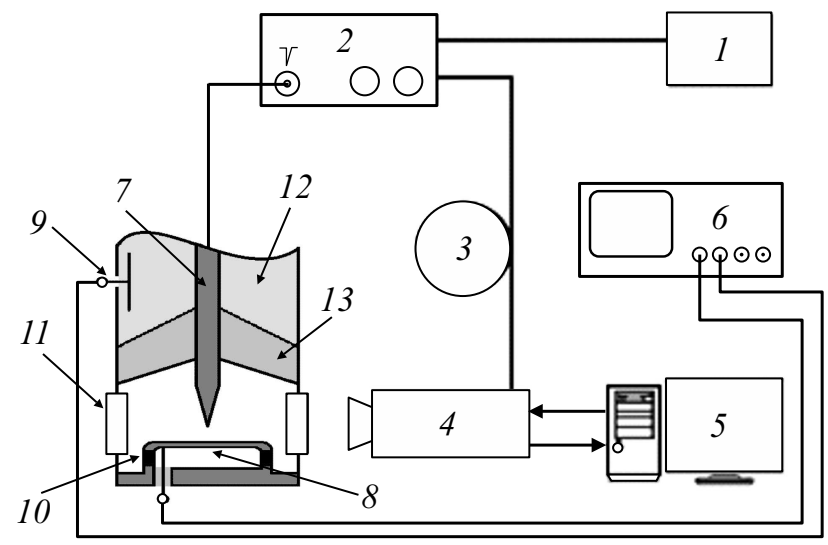

Рис. 1. Блок-схема экспериментальной установки. 1 - генератор запуска, 2 - генератор импульсов напряжения. 3 - линия задержки (ICCD-камера) либо генератор задержек (стриккамера), 4 - четырехканальная ICCD-камера либо стриккамера, 5 - компьютер, 6 - осциллограф, 7 - высоковольтный электрод, $8-$ плоский заземленный электрод, 9 - емкостной делитель напряжения, 10 - чип-резисторы, 11 - окно, 12 - трансформаторное масло, 13 - изолятор (капролон).

при различном давлении газов (аргон, азот) и обеих полярностях импульса напряжения.

\section{Экспериментальная установка и методики}

Эксперименты проводились на двух установках, блоксхема которых представлена на рис. 1. Первая установка (Установка 1) включала в себя разрядную камеру, два генератора импульсов напряжения отрицательной (ГИН-100-1, $\left.U \approx 25 \mathrm{kV}, \tau_{0.5} \approx 3 \mathrm{~ns}, \tau_{0.1-0.9} \approx 0.7 \mathrm{~ns}\right)$ и положительной (ГИН-50-1, $U \approx 25 \mathrm{kV}, \tau_{0.5} \approx 10 \mathrm{~ns}$, $\left.\tau_{0.1-0.9} \approx 2 \mathrm{~ns}\right)$ полярности, разработанных по технологии FID (компания „Антарес“, Санкт-Петербург), и четырехканальную ICCD (Intersified Crarge Coupled Device - прибор с зарядовой связью, оснащенный усилителем) камеру HSFC PRO (минимальна длительность экспозиции - $3 \mathrm{~ns}$ ). Вторая установка (Установка 2) включала в себя разрядную камеру, генератор импульсов напряжения отрицательной полярности (ГИН-55-01, $U \approx 55 \mathrm{kV}, \tau_{0.5} \approx 1 \mathrm{ns,}$ $\tau_{0.1-0.9} \approx 0.7 \mathrm{~ns}$, „Антарес“, Санкт-Петербург) и стриккамеру Hamamatsu C10910-05, оснащенную монохроматором Acton SpectraPro SP-2300 (Princeton Instruments).

На обеих установках использовалась одна и та же газоразрядная камера, конструкция которой подробно описана в работах $[17,19,20]$. Высоковольтный электрод имел форму конуса с диаметром основания $6 \mathrm{~mm}$ и углом раствора при вершине конуса $30^{\circ}$, а заземленный - плоскую форму. На установке 2 в качестве высоковольтного электрода также применялся трубчатый 
электрод диаметром $6 \mathrm{~mm}$ с острой кромкой. Высоковольтные электроды изготавливались из алюминия или нержавеющей стали. Межэлектродное расстояние $d$ на установке 1 составляло $3 \mathrm{~mm}$, а на установке 2 менялось от 3 до $5 \mathrm{~mm}$.

Газоразрядная камера оснащена емкостным делителем напряжения, токовым шунтом, изготовленным из чипрезисторов, и коллектором для регистрации убегающих электронов (диаметр приемной части коллектора $16 \mathrm{~mm}$, временное разрешение - $60 \mathrm{ps}$ ).

Запуск каналов ICCD-камеры на установке 1 осуществлялся на 2-3 ns раньше момента прихода импульса напряжения на промежуток. Это позволило осуществить регистрацию свечения плазмы в предпробойной стадии разряда, длительность которой в условиях эксперимента составляла менее 2 ns. Благодаря наличию джиттера по всем четырем каналам ICCD-камеры и набору статистики ( $\sim 10^{2}$ реализации) удалось восстановить процесс продвижения стримера по промежутку. Необходимо отметить, что в этом случае отсутствует временна́я привязка полученных изображений к импульсам напряжения.

Посредством стрик-камеры на установке 2 осуществлялась регистрация временного хода интенсивности излучения колебательно-вращательной полосы второй положительной системы азота на длине волны $337.1 \mathrm{~nm}$. Регистрация излучения осуществлялась из различных зон вдоль промежутка. Размер зоны по длине промежутка составлял $250 \mu \mathrm{m}$, а по его ширине - $4 \mathrm{~mm}$. Итоговая осциллограмма временного хода интенсивности излучения в каждой зоне получалась усреднением по 300 импульсам. По полученным данным оценивалась скорость продвижения фронта стримера.

В отдельной серии экспериментов осуществлялась регистрация импульсов напряжения, тока разряда и тока пучка убегающих электронов (сверхкороткий лавинный электронный пучок - СЛЭП [20]). Условия эксперимента (род газа, давление, межэлектродное расстояние, напряжение на выходе ГИН) были такими же, как в экспериментах с ICCD- и стриккамерами. При регистрации убегающих электронов в качестве плоского заземленного электрода использовалась алюминиевая фольга толщиной $10 \mu \mathrm{m}$. Сигналы с емкостного делителя напряжения, токового шунта и коллектора регистрировались цифровыми осциллографами LeCroy WaveMaster 830Zi-A (полоса пропускания - $30 \mathrm{GHz}$, шаг дискретизации - $12.5 \mathrm{ps}$ ) и Agilent DSO-X6004A (полоса пропускания - $6 \mathrm{GHz}$; шаг дискретизации - $50 \mathrm{ps}$ ). При этом одновременно могли регистрироваться либо напряжение и ток разряда, либо напряжение и ток СЛЭП. Аналогичные исследования параметров СЛЭП проводились в работах $[1,7,9,14,20,21,23,27]$.

На установке 1 газоразрядная камера заполнялась азотом либо аргоном, а на установке 2 - только азотом. Давление газов менялось в диапазоне $12.5-400 \mathrm{kPa}$.

\section{Экспериментальные результаты}

В ходе экс периментов было установлено, что в промежутке всегда формируется диффузный (объемный) разряд при обеих полярностях. На рис. 2 приведены интегральные по времени фотографии свечения плазмы разряда в аргоне и азоте, полученные с помощью цифрового фотоаппарата SONY A100. На промежуток подавались импульсы напряжения наносекундной длительности от генератора ГИН-55-01 (установка 2). Диффузный характер разряда обусловлен формированием в условиях высокого перенапряжения стримера большого размера. Образование искровых каналов могло произойти при давлении газов более $200 \mathrm{kPa}$ и только при повторном пробое, вызванном приходом на промежуток через каждые $30 \mathrm{~ns}$ отраженных импульсов напряжения. Наличие отраженных импульсов обусловлено отсутствием согласования импедансов нагрузки (газоразрядный промежуток) и генератора.

B экспериментах c ICCD-камерой на установке 1 исследовалось влияние рода газа, давления и полярности импульса напряжения на размеры стримера, а также определялось место инициирования разряда. На рис. 3-5 приведены изображения свечения плазмы на различных этапах формирования стримера и прохождения им промежутка. Эти данные получены на установке 1 при разряде в аргоне давлением 12.5, 100 и $400 \mathrm{kPa}$ и при обеих полярностях. На рис. 6 и 7 представлены соответствующие осциллограммы. Отметим, что амплитуда напряжения в падающей волне была одной и той же при обеих полярностях - $\approx 25 \mathrm{kV}$.

Было установлено, что при давлении аргона $12.5-100 \mathrm{kPa}$ свечение плазмы появляется на расстоянии $\sim 1 \mathrm{~mm}$ от вершины острийного электрода, а не вблизи нее, как ожидалось (рис. 3). Это можно объяснить уменьшением коэффициента ударной ионизации $\alpha$ при высоких значениях приведенной напряженности электрического поля $E / p$ вблизи острия. Расчеты показывают, что для данного разрядного промежутка при амплитуде напряжения $25 \mathrm{kV}$ напряженность электрического поля вблизи острия достигает $\sim 1 \mathrm{MV} / \mathrm{cm}$. Из рис. 3 видно, что и на последующих этапах формирующаяся плазма имеет форму, близкую к шару, размеры которого постепенно увеличиваются до замыкания промежутка. После этого на катоде появляется пятно, яркость свечения которого нарастает по мере роста тока разряда.

При увеличении давления аргона $(100 \mathrm{kPa}$ и более) точка, в которой появляется свечение/плазма, постепенно смещается к вершине острия. На последующих этапах также наблюдается распространение стримера, размер которого увеличивается по мере его продвижения к плоскому электроду.

Из полученных данных следует, что размер стримера уменьшается с ростом давления газа. Кроме того, при давлении $400 \mathrm{kPa}$ иногда наблюдаются два стримера с меньшими поперечными размерами. После замыкания 


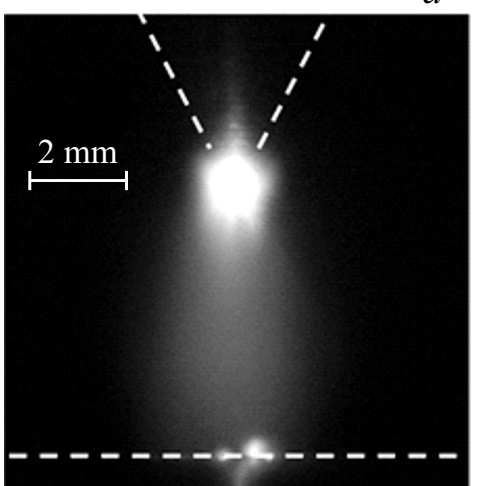

b

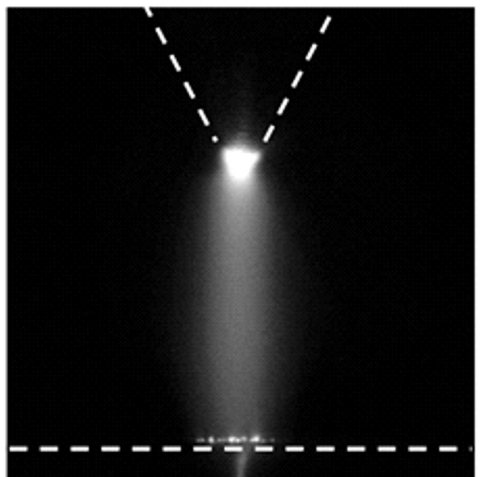

C

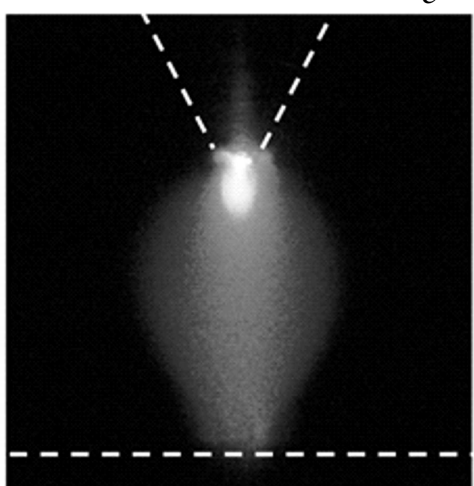

Рис. 2. Фотографии свечения плазмы разряда в аргоне при давлениях $p=25(a)$ и $200 \mathrm{kPa}(b)$, а также в азоте при $p=300 \mathrm{kPa}(c)$ при отрицательной полярности острийного электрода (вверху). Генератор ГИН-55-01. Длительность экспозиции - $1 \mathrm{~s}$.
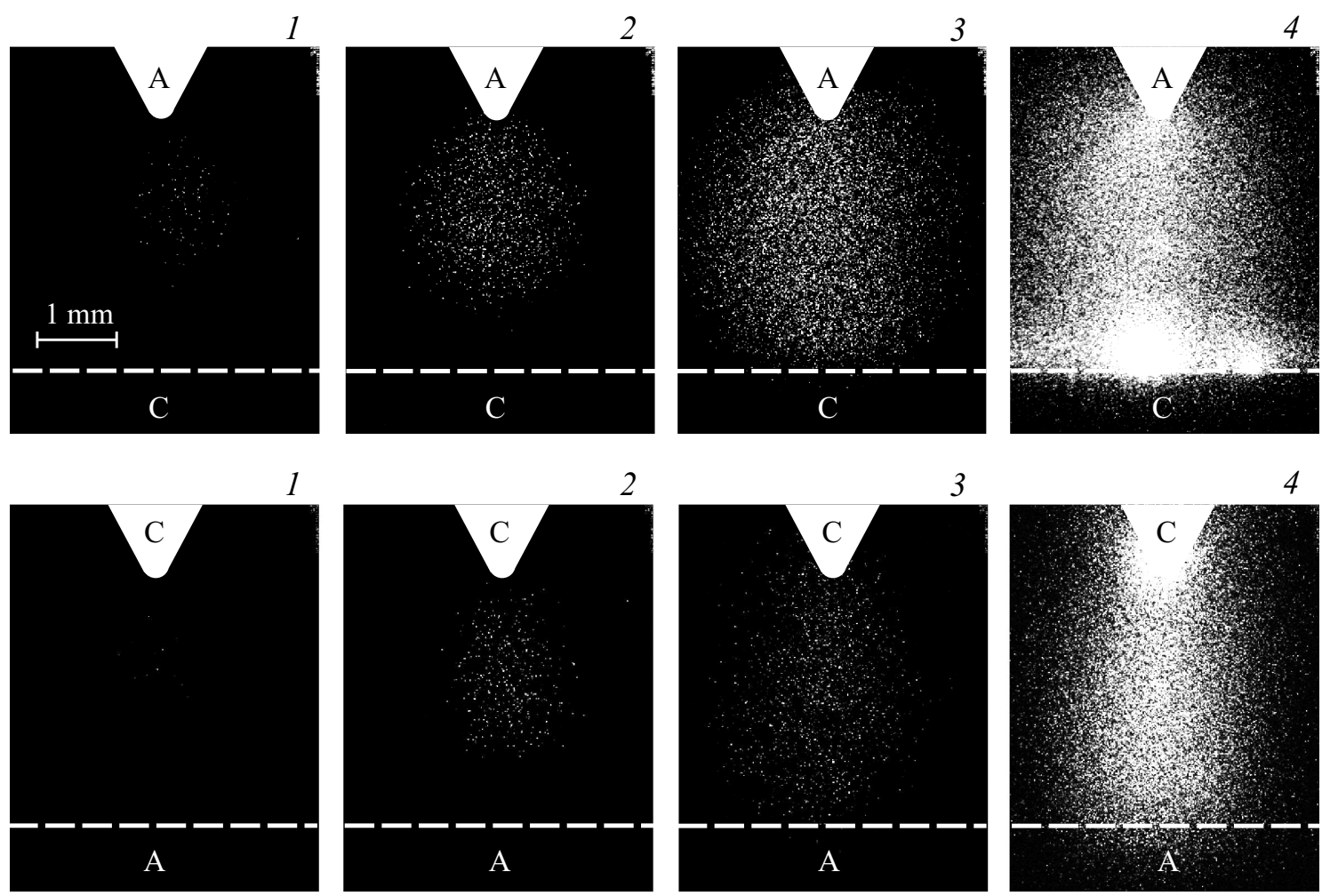

Рис. 3. Изображения свечения плазмы в различные моменты времени. Давление аргона - $12.5 \mathrm{kPa}$. Верхний ряд - положительная полярность (ГИН-50-1), нижний - отрицательная полярность (ГИН-100-1). А — анод, С - катод.

промежутка плазмой на катоде образуется пятно. При давлении 200-400 kРа пятно появляется как на катоде, так и на аноде.

Полярность импульса напряжения заметно не повлияла на динамику развития стримера. Отметим, что длительность фронта импульса напряжения при положительной полярности была в три раза больше, чем при отрицательной.

В азоте при тех же давлениях и обеих полярностях наблюдалась аналогичная динамика формирования разряда. Подобная форма стримера также наблюдалась в экспериментах, описанных в работах $[3,11,24]$. Моделирование стримера $[26,29]$ в условиях субнаносекундного пробоя показало, что размер стримера достигает $\sim 1 \mathrm{~cm}$ при $d=1.5 \mathrm{~cm}$.

Скорость стримера оценивалась по данным, полученным с помощью стрик-камеры на установке 2. Оценка осуществлялась по задержке появления свечения близи плоского электрода относительно острийного. Началом свечения плазмы служил уровень сигнала в $2 \%$ от максимума. Так, средняя скорость стримера при давлении азота $100 \mathrm{kPa}$ составила $1.8 \mathrm{~cm} / \mathrm{ns}$. На рис. 8 показан ход 

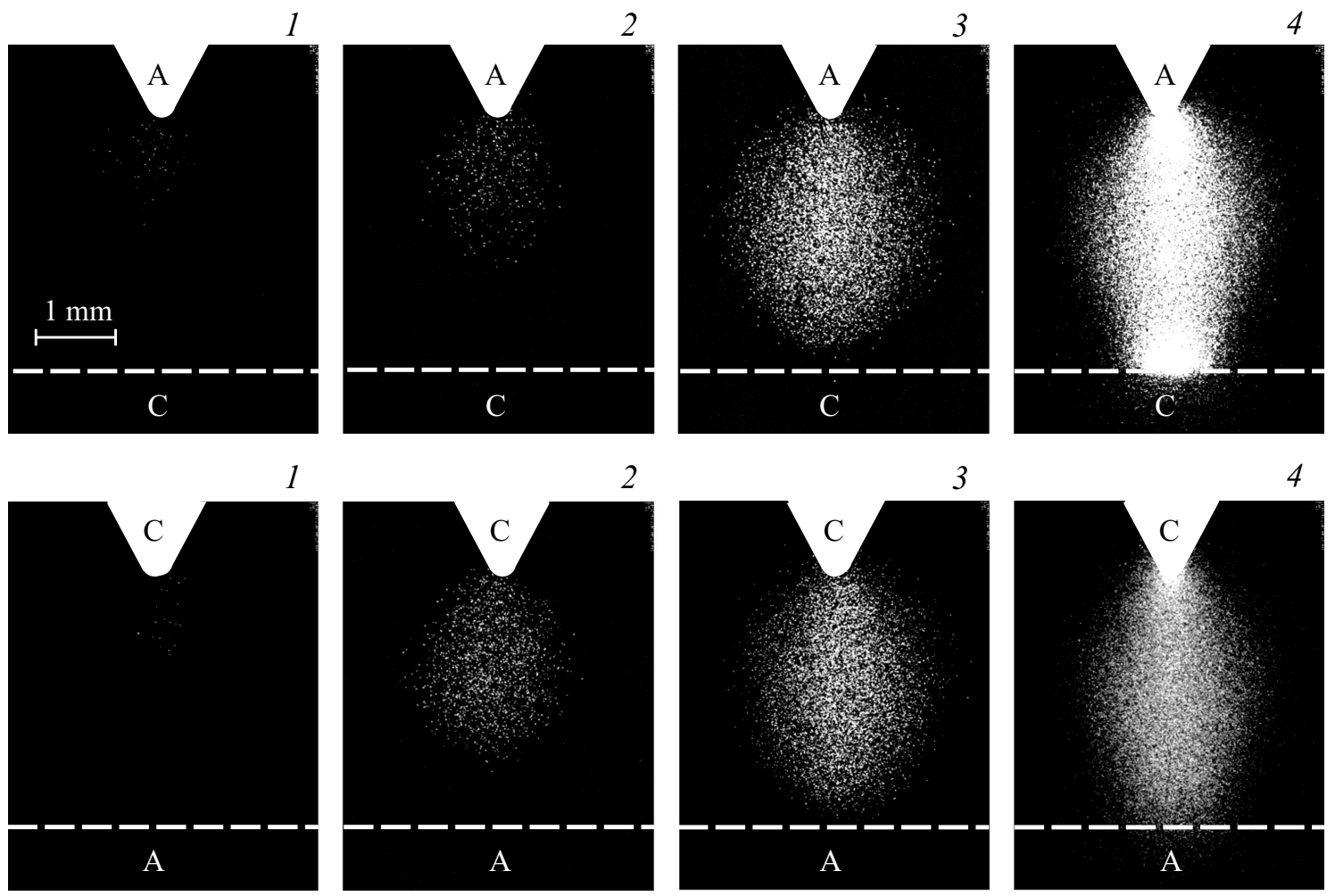

2
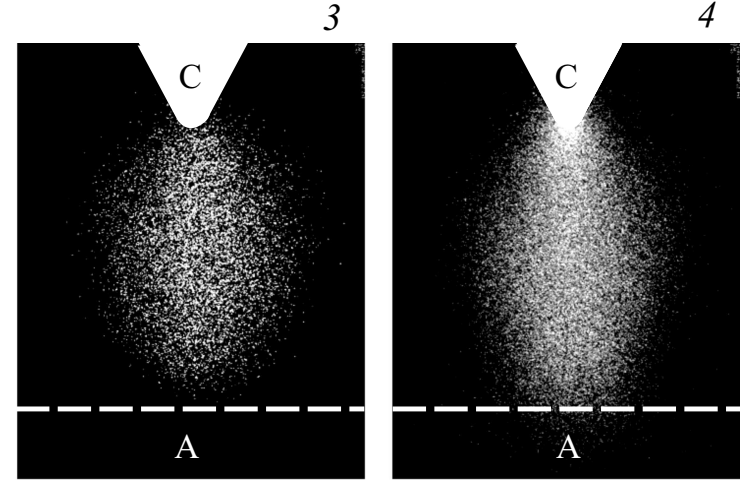

Рис. 4. Изображения свечения плазмы в различные моменты времени. Давление аргона $-100 \mathrm{kPa}$. Верхний ряд - положительная полярность (ГИН-50-1), нижний — отрицательная полярность (ГИН-100-1). А — анод, С — катод.
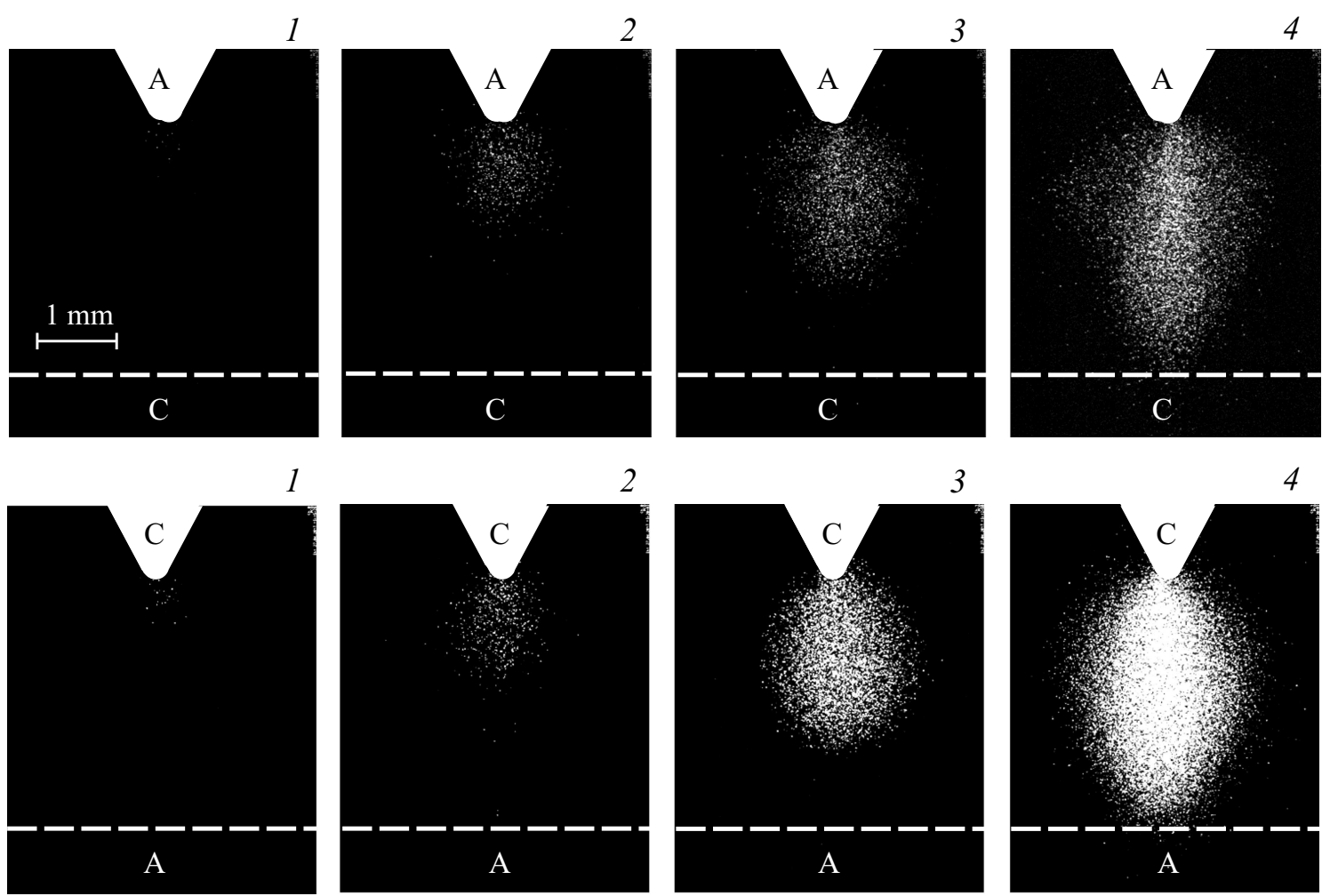

Рис. 5. Изображения свечения плазмы в различные моменты времени. Давление аргона - $400 \mathrm{kPa}$. Верхний ряд - положительная полярность (ГИН-50-1), нижний — отрицательная полярность (ГИН-100-1). А — анод, С — катод. 
во времени интенсивности излучения в различных зонах промежутка. Из этих данных следует, что скорость распространения фронта плазмы до середины промежутка меньше, чем от середины до плоского электрода. Это совпадает с данными, полученными в работах [21,33], в которых свечение из различных зон разрядного промежутка регистрировалось с помощью фотодиода с временным разрешением $\approx 80 \mathrm{ps}$. С уменьшением давления скорость стримера обычно увеличивалась.

При отрицательной полярности импульсов напряжения в широком диапазоне давлений за плоским электродом из алюминиевой фольги толщиной $10 \mu \mathrm{m}$ регистрировался СЛЭП, в частности, при напряжении $U \approx-55 \mathrm{kV}$ СЛЭП регистрировался при давлении азота $300 \mathrm{kPa}$. Как и в предыдущих наших работах $[1,20,21,23]$, СЛЭП регистрировался вблизи максимума напряжения, на нарастающей части тока разряда.

\section{Обсуждение результатов}

Из рис. 3-5 следует, что область свечения плазмы в промежутке увеличивается со временем до прихода
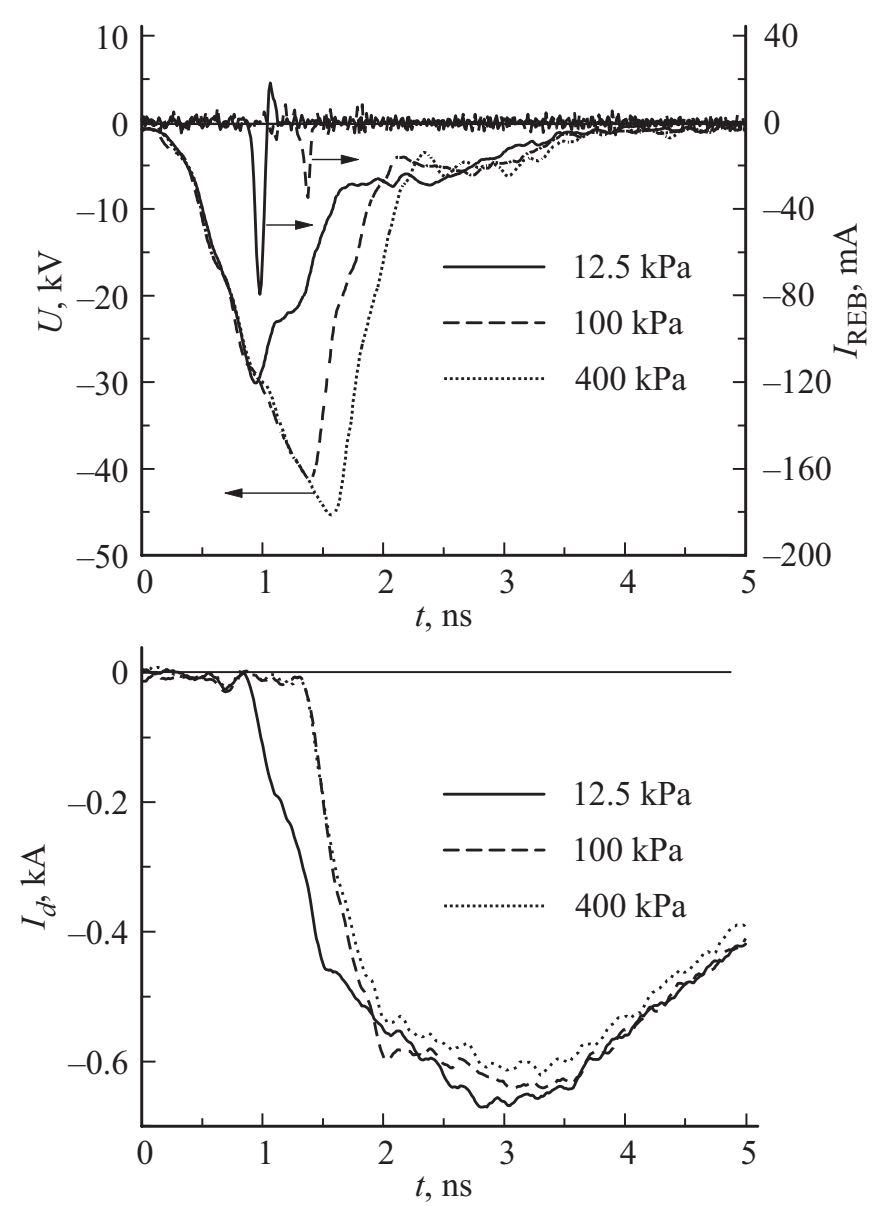

Рис. 6. Осциллограммы импульсов напряжения $U$, тока разряда $I_{d}$ и тока пучка убегающих электронов $I_{R E B}$ в аргоне при различном давлении. ГИН-100-1. Установка 1. Осциллограф LeCroy WaveMaster 830Zi-A, $30 \mathrm{GHz}$.
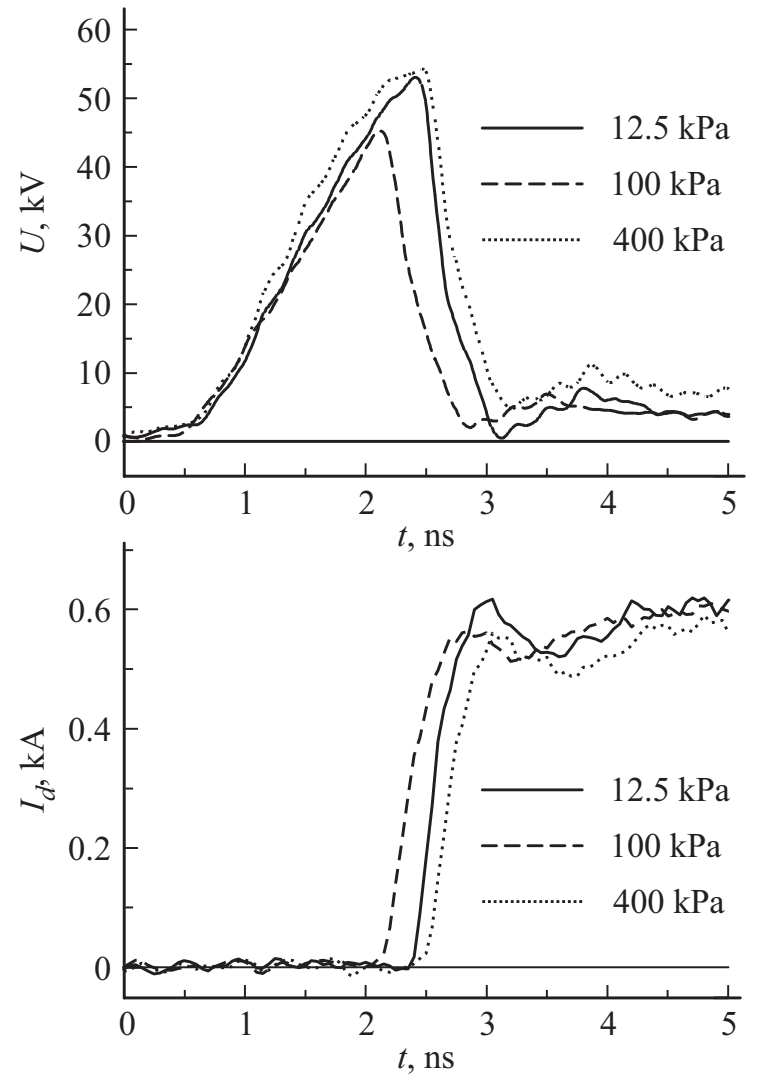

Рис. 7. Осциллограммы импульсов напряжения $U$ и тока разряда $I_{d}$ в аргоне при различном давлении. ГИН-50-1. Установка 1. Осциллограф Agilent DSO-X6004A, $6 \mathrm{GHz}$.

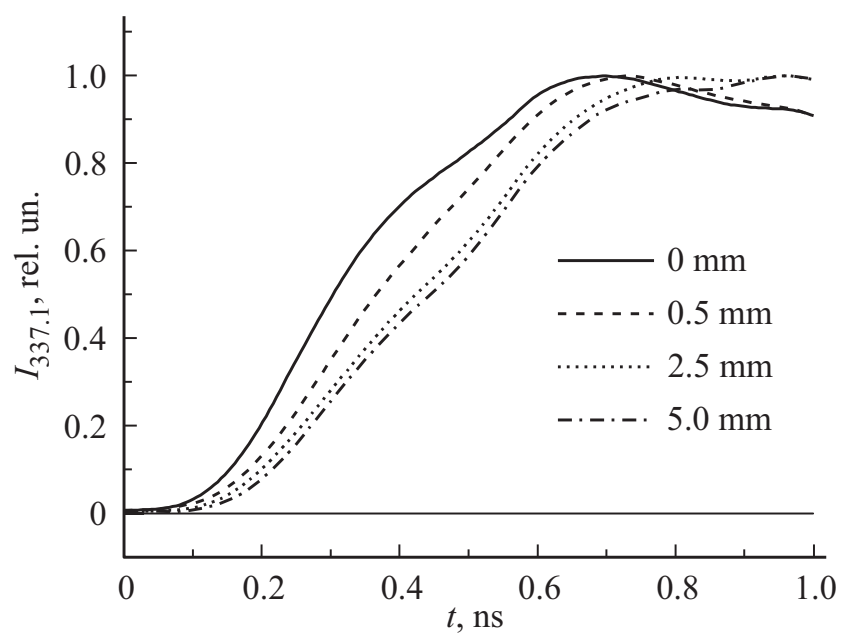

Рис. 8. Временной ход интенсивности излучения $I_{337.1}$ полосы $337.1 \mathrm{~nm}$ молекулы азота на различных расстояниях от катода. Давление азота - $0.1 \mathrm{MPa}$. Промежуток „трубкаплоскость“. Межэлектродное расстояние $d=5 \mathrm{~mm}$. Установка 2.

фронта плазмы к плоскому электроду. По-видимому, при отрицательной полярности убегающие электроны могут генерироваться не только в сторону плоского анода, но 
также и в боковом направлении. Это предположение сделано исходя из геометрии плазменного образования: плазма имеет форму, близкую к шару, увеличивающемуся в размерах. Именно на фронте плазмы, где напряженность электрического поля высока и достигает критического значения, часть электронов может перейти в режим непрерывного ускорения. Формирование убегающих электронов, как следует из расчетов [22], продолжается до достижения фронтом плазмы плоского анода.

В работах [1,34] убегающие электроны в боковом направлении были зарегистрированы. В экспериментах использовалась газоразрядная камера, оснащенная двумя коллекторами. Один коллектор располагался за плоским электродом из фольги, другой - сбоку в специальном отверстии, позволяющем установить фольгу. На промежуток подавались импульсы напряжения амплитудой $\approx 200 \mathrm{kV}$. Амплитуда тока пучка убегающих электронов, зарегистрированного в боковом направлении, была меньше, чем в прямом, но длительность импульсов существенно не отличалась.

Согласно [30,35], часть электронов с энергией 0.1-1 keV, генерирующаяся на фронте плазмы, не может перейти в режим непрерывного ускорения, поскольку эти значения энергии соответствуют максимальным значениям ионизационных потерь энергии электроном. Так, например, максимум сечения ионизации молекулы азота с образованием молекулярного иона соответствует энергии электрона $100 \mathrm{eV}$ [36]. При этом „хвост“ сечения ионизации тянется до значений энергии $\sim 1 \mathrm{keV}$ и более [36].

Вероятно, электроны с энергией $0.1-10 \mathrm{keV}$ обеспечивают предварительную ионизацию газа перед фронтом плазмы при отрицательной полярности импульса напряжения. Такие электроны будем называть быстрыми, а электроны, способные набрать энергию $\geq 10 \mathrm{keV}$, убегающими. Быстрые электроны обеспечивают продвижение стримера к аноду со скоростью $>1 \mathrm{~cm} / \mathrm{ns}$, а также увеличение его поперечных размеров.

При положительной полярности острийного электрода форма стримера существенно не отличается от формы стримера при отрицательной полярности. Мы считаем, что при положительной полярности предварительная ионизация газа осуществляется квантами характеристического рентгеновского излучения [33], генерирующимися при взаимодействии быстрых электронов с атомами и молекулами газа. Появившиеся в результате этого процесса начальные электроны становятся быстрыми, поскольку попадают в область с высокой напряженностью электрического поля на фронте плазмы. Быстрые электроны обеспечивают высокую скорость ионизации газа и, как следствие, высокую скорость перемещения фронта плазмы.

В работе [33] при моделировании было показано, что интенсивность характеристического рентгеновского излучения возрастает с уменьшением атомного номера газа, заполняющего промежуток. Кроме того, характеристическое рентгеновское излучение экспериментально было зарегистрировано в азоте при положительной полярности острийного электрода [25].

Отметим, что в начале пробоя предварительная ионизация газа осуществляться тормозным рентгеновским излучением, возникающим при торможении первых быстрых электронов анодом.

По мере роста давления газа величина $E / p$ становится меньше. В результате область пространства, в которой $\alpha$ достигает наибольших значений, смещается к вершине острийного электрода, и мы видим, что свечение плазмы при высоком давлении газа появляется не вдали от острийного электрода, как при низком давлении газа (рис. 3), а вблизи его вершины (рис. 4 и 5). Кроме того, при высоком давлении газа, а также с учетом распределения напряженности электрического поля в промежутке при наличии плазмы значение величины $E / p$ на боковой поверхности стримера может быть недостаточно для ускорения электронов до высоких энергий. В результате количество быстрых электронов с ростом давления газа убывает, эффективность предварительной ионизации газа в боковом направлении становится меньше, темпы ионизации падают. Тем не менее в направлении распространения стримера значение величины $E / p$ на его фронте может быть большим и превышать критическое значение для убегания электронов. Таким образом, темпы ионизации в направлении плоского электрода выше, чем в боковом, и мы видим стример с меньшими поперечными размерами. В настоящей работе при отрицательной полярности импульса напряжения пучок убегающих электронов с $U \approx-55 \mathrm{kV}$ был зарегистрирован при давлении азота $300 \mathrm{kPa}$.

\section{Выводы}

Проведенные исследования показали, что при подаче наносекундных импульсов напряжения на промежуток „острие-плоскость“ в азоте и аргоне при давлениях $12.5-400 \mathrm{kPa}$ формируется диффузный разряд. Посредством ICCD-камеры было установлено, что при относительно низком для условий данного эксперимента давлении газов (до $50 \mathrm{kPa}$ ) плазма зарождается на некотором расстоянии ( $1 \mathrm{~mm}$ в условиях эксперимента) от вершины острийного электрода. При этом плазменное образование имеет форму, близкую к шару, диаметр которого увеличивается до перемыкания промежутка плазмой и может достигать величины порядка $d$.

По мере роста давления газов $(100 \mathrm{kPa}$ и более) зона инициирования разряда смещается к вершине острийного электрода. При этом стример развивается от острийного электрода и на начальном этапе также имеет форму шара. Установлено, что поперечные размеры стримера уменьшаются с ростом давления газов.

Уменьшение размеров стримера с ростом давления газов можно объяснить уменьшением значения величи- 
ны $E / p$ на боковой поверхности стримера, что приводит к уменьшению числа электронов с высокими энергиями и, как следствие, эффективности предварительной ионизации газа в боковом направлении. При этом в направлении распространения стримера $E / p$ превышает критическое значение для убегания электронов, что подтверждается регистрацией СЛЭП при высоком (300 kPa) давлении азота.

Предполагается, что предварительную ионизацию газа перед стримером при отрицательной полярности острийного электрода осуществляют быстрые электроны с энергией $0.1-10 \mathrm{keV}$, а при положительной кванты характеристического рентгеновского излучения. При этом высокие темпы ионизации газа при обеих полярностях обеспечиваются быстрыми электронами, которые получают энергию в высоком электрическом поле на фронте плазмы. Это обусловливает отсутствие существенных отличий в размерах стримера при отрицательной и положительной полярностях.

Работа выполнена за счет гранта Российского научного фонда, проект № 17-72-20072.

\section{Список литературы}

[1] Tarasenko V.F., Baksht E.Kh., Burachenko A.G., Kostyrya I.D., Lomaev M.I., Rybka D.V. // Plasma Devices and Operation. 2008. Vol. 16. N 4. P. 267-298.

[2] Naidis G.V. // Phys. Rev. E. 2009. Vol. 79. N 5. P. 57401.

[3] Tardiveau P., Moreau N., Bentaleb S., Postel C., Pasquiers S. // J. Phys. D: Appl. Phys. 2009. Vol. 42. N 17. P. 175202.

[4] Babaeva N.Yu., Kushner M.J. // Plasma Sources Sci. Technol. 2009. Vol. 18. N 3. P. 035009.

[5] Костыря И.Д., Тарасенко В.Ф. // ЖТФ. 2010. Т. 80. Вып. 2. C. 111-117.

[6] Pai D.Z., Stancu G.D., Lacoste D.A., Laux C.O. // Plasma Sources Sci. Technol. 2009. Vol. 18. N 4. P. 045030.

[7] Тарасенко В.Ф., Бакшт Е.Х., Бураченко А.Г., Костыря И.Д., Ломаев М.И., Рыбка Д.В. // ЖТФ. 2010. Т. 80. Вып. 2. С. 51-59.

[8] Ivanov S.N., Lisenkov V.V., Shpak V.G.// J. Phys. D: Appl. Phys. 2010. Vol. 43. N 31. P. 315204.

[9] Tarasenko V.F., Baksht E.Kh., Shut'ko Yu.V. // IEEE Trans. Plas. Sci. 2011. Vol. 39. N 11. P. 2096-2097.

[10] Yatom S., Vekselman V., Gleizer J.Z., Krasik Ya.E. // J. Appl. Phys. 2011. Vol. 109. N 7. P. 073312.

[11] Starikovskiy A.Yu. // IEEE Trans. Plas. Sci. 2011. Vol. 39. N 11. P. 2602-2603.

[12] Shao T., Tarasenko V.F., Zhang Ch., Baksht E.Kh., Yan P., Shut'ko Yu.V. // Laser and Particle Beams. 2012. Vol. 30. N 3. P. 369-378.

[13] Levko D., Krasik Ya.E., Tarasenko V.F. // Int. Rev. Phys. 2012. Vol. 6. N 2. P. 165-195.

[14] Бойченко А.М., Тарасенко В.Ф., Бакшт Е.Х., Бураченко А.Г., Ерофеев М.В., Ткачев А.Н. // ЖТФ. 2013. Т. 83. Вып. 3. С. 61-65.

[15] Shao T., Tarasenko V.F., Zhang Ch., Burachenko A.G., Rybka D.V., Kostyrya I.D., Lomaev M.I., Baksht E.Kh., Yan P. // Rev. Sci. Instrum. 2013. Vol. 84. N 5. P. 053506.
[16] Ерофеев М.В., Бакит Е.Х., Тарасенко В.Ф., Шутько Ю.В. // ЖТФ. 2013. Т. 83. Вып. 2. С. 52-58.

[17] Тарасенко В.Ф., Бакит Е.Х, Бураченко А.Г., Ерофеев М.В., Ломаев М.И. // ЖТФ. 2014. Т. 84. Вып. 4. С. 26-30.

[18] Naidis G.V. // IEEE Trans. Dielectr. Electr. Insul. 2015. Vol. 22. N 5. P. $2428-2432$.

[19] Бакшт Е.X, Бураченко А.Г., Ерофеев М.В., Тарасенко В.Ф. // Физика плазмы. 2014. Т. 40. Вып. 5. С. 480-488.

[20] Генерация убегающих электронов и рентгеновского излучения в разрядах повышенного давления / Под ред. В.Ф. Тарасенко. Томск: STT, 2015. 568 с.

[21] Тарасенко В.Ф., Белоплотов Д.В., Ломаев М.И. // Физика плазмы. 2015. Т. 41. Вып. 10. С. 902-917.

[22] Kozhevnikov V.Yu., Kozyrev A.V., Semeniuk N.S. // Europhys. Lett. 2015. Vol. 112. N 15. P. 15001.

[23] Beloplotov D.V., Tarasenko V.F., Lomaev M.I., Sorokin D.A. // IEEE Trans. Plas. Sci. 2015. Vol. 43. N 11. P. 3808-3814.

[24] Tardiveau P., Magne L., Marode E., Ouaras K., Jeanney P., Bournonville B. // Plasma Sources Sci. Technol. 2016 Vol. 25. N 5. P. 054005.

[25] Тарасенко В.Ф., Бакшт Е.Х., Бураченко А.Г., Ломаев М.И. // Прикладная физика. 2016. Вып. 4. С. 49-53.

[26] Babaeva N.Yu., Tereshonok D.V., Naidis G.V. // Plasma Sources Sci. Technol. 2016. Vol. 25. N 4. P. 044008.

[27] Tarasenko V.F., Baksht E.Kh., Beloplotov D.V., Burachenko A.G., Lomaev M.I., Sorokin D.A. // Laser and Particle Beams. 2016. Vol. 34. N 4. P. 748-763.

[28] Babich L.P., Loiko T.V. // IEEE Trans. Plasma Sci. 2016. Vol. 44. N 12. P. 3243-3248.

[29] Babaeva N.Yu., Naidis G.V. // Phys. Plasmas. 2016. Vol. 23. N 8. P. 083527.

[30] Byszewski W.W., Reinhold G. // Phys. Rev. A. 1982. Vol. 26. N 5. P. $2826-2831$.

[31] Яковленко С.И. // ЖТФ. 2004. Т. 74. Вып. 9. С. 47-54.

[32] Lomaev M.I., Beloplotov D.V., Tarasenko V.F., Sorokin D.A. // IEEE Trans. Dielectr. Electr. Insul. Vol. 22. N 4. P. 1833-1840.

[33] Козырев А.В., Тарасенко В.Ф., Бакшт Е.Х., Шутько Ю.В. // Письма в ЖТФ. 2011. Т. 37. Вып. 22. С. 26-33.

[34] Baksht E.Kh., Kostyrya I.D., Lomaev M.I., Rybka D.V., Tarasenko V.F. // Physics of Wave Phenomena. 2008. Vol. 16. N 3. P. 199-206.

[35] Kunhardt E.E., Byszewski W.W. // Phys. Rev. A. 1980. Vol. 21. N 6. P. 2069-2077.

[36] Itikawa Y. // J. Phys. and Chem. Reference Data. 2006. Vol. 31. N 31. P. 31-3. 